Case Report

\title{
Histoplasmosis in Sri Lanka - a masquerader in a strange land: A Case Report
}

\author{
MN Jayawardena ${ }^{1}$, K. Siriwardane ${ }^{2}$, P. Jayasooriya ${ }^{3}$, S Sigera $^{1}$, \\ PI Jayasekera ${ }^{1}$ \\ Sri Lankan Journal of Infectious Diseases 2019 Vol.9 (2):152-155 \\ DOI: http://doi.org/10.4038/sljid.v9i2.8242
}

\begin{abstract}
We present a patient with an oral mucosal ulcer who was diagnosed with disseminated histoplasmosis. This fungal infection is endemic in the United States, and thus may be not considered in the differential diagnosis of oral ulcers in Sri Lanka. Furthermore, it may mimic many common diseases found in Sri Lanka. It is therefore important to be aware of this entity, since it is potentially curable if diagnosed and treated early.
\end{abstract}

Key Words: Histoplasmosis, Sri Lanka, Histoplasma capsulatum

\section{Introduction}

Histoplasma capsulatum is a soil inhabiting fungus, which causes infections most often in the immunocompromised host. Most cases are reported in the Midwest and Southeast United States, but Sri Lanka is by no means free of this disease. This fungus is acquired by inhalation and classically forms caseating and non-caseating granulomas. It is important to consider this entity, as it mimics many common diseases, and is potentially curable if diagnosed and treated early. Here, we report a case of probable disseminated histoplasmosis, diagnosed in early 2017.

\section{Case history}

A 66-year-old retired labourer presented to the Oromaxillary Facial Unit of Teaching Hospital Kegalle, complaining of an oral ulcer of six-months duration (Figure 1). He was a diabetic, well-

${ }^{1}$ Department of Mycology, Medical Research Institute, Colombo 8, Sri Lanka

${ }^{2}$ Oromaxillary Facial Unit, Teaching Hospital, Kegalle, Sri Lanka

${ }^{3}$ Department of Oral Pathology, Faculty of Dental Sciences, University of Peradeniya, Sri Lanka

Address for correspondence: Dr. Naamal Jayawardena, Department of Mycology, Medical Research Institute, Colombo 8, Sri Lanka. Telephone: +94773074599 Email: naamaljay@gmail.com

(iD) https://orcid.org/0000-0003-1928-8086

Received 9 April 2019 and revised version accepted 20 July 2019

(c) (7) use, distribution, and reproduction in any medium, provided the original author and source are credited. 
controlled on oral-hypoglycemics, a smoker and betel chewer. During this period, he also complained of low-grade fever, dysphagia and weight loss. There was a pus discharging mucosal ulcer in the mid palatal region extending to the palatal aspect of the left anterior maxilla. Systemic examination was normal and there was no cervical

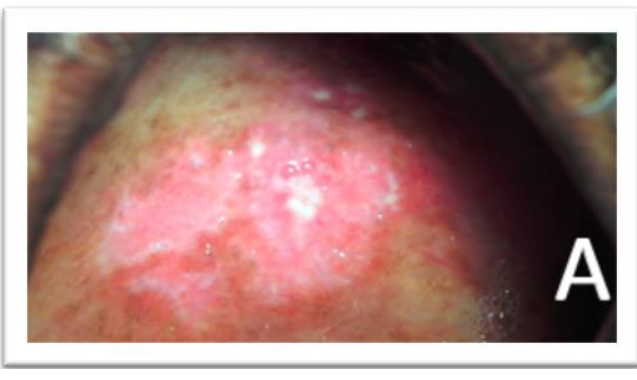

Figure 1: Oral ulcer in upper palate at initial presentation lymphadenopathy. Full blood counts and liver function tests were normal. Moderately elevated renal functions and an elevated sedimentation rate $\left(55 \mathrm{~mm} 1^{\text {st }}\right.$ hour$)$ were detected.

Samples following incisional biopsy of the alveolar ridge were dispatched for histology, suspecting oral squamous cell carcinoma. The histology, however, showed presence of yeast forms, which, following fungal staining, was suggestive of histoplasmosis (Figure 2). Fungal studies confirmed the presence of

Histoplasma capsulatum, with yeast forms seen in the direct smear (Figure 3) and thermal dimorphism demonstrated on culture. The patient was started on oral itraconazole 200mg twice daily, with monitoring of liver enzymes. He showed good response, with the oral ulcer regressing over time.

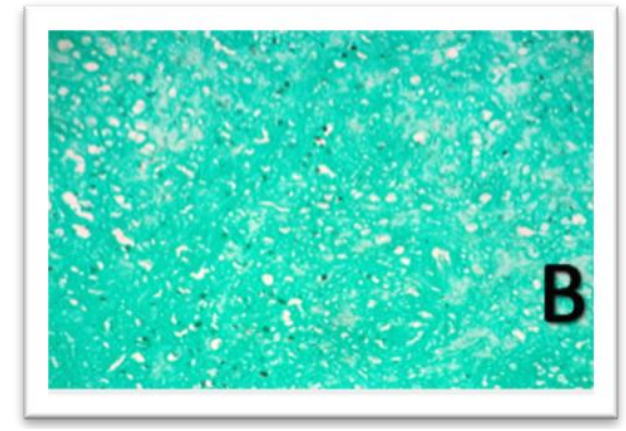

Figure 2: Histoplasma yeast forms at microscopy x20 stained with Grocott methanamine

His retroviral screening was negative. As the chest $\mathrm{X}$ ray, ultrasound scan and non-contrast CT chest revealed multiple minute opacities in bilateral lung fields, the patient was referred to a chest physician, even though the patient didn't complain of any respiratory symptoms. Bronchoscopy was performed and brushing and washing samples were sent for fungal studies. The direct smear revealed yeast forms, but cultures were sterile.

After completion of 6 weeks of itraconazole therapy, the patient's oral ulcers resolved with no significant drug-related adverse effects. However, the patient passed away from unrelated causes a few months later. The timeline of the patient's course is shown in Figure 4. 


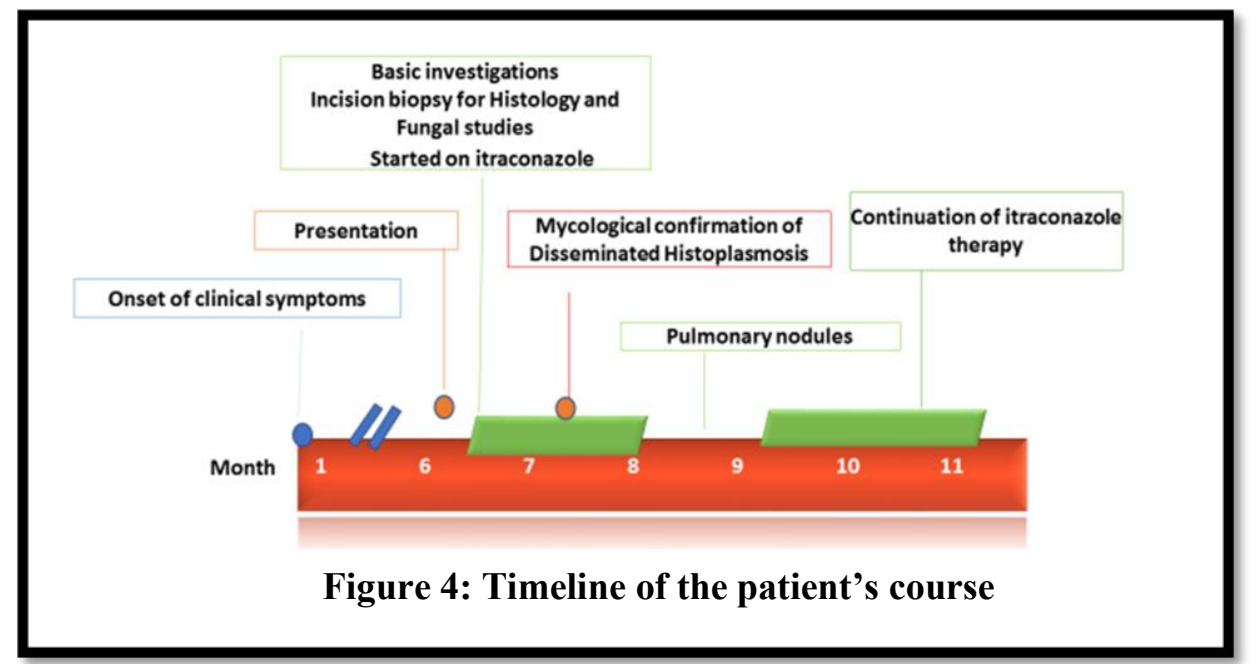

\section{Discussion}

Histoplasmosis has been documented worldwide ${ }^{1}$, while Sri Lanka first reported it in $1975 .^{2}$ It has previously been found that $4 \%$ of healthy adults and $6.3 \%$ of those with chronic lung disease in Sri Lanka test positive for the histoplasma skin test. ${ }^{3}$ So far, five indigenous cases of histoplasmosis have been reported in Sri Lanka. ${ }^{2-7}$ Four of these patients presented with oral ulcers and the other presented with features of disseminated histoplasmosis with hepatosplenomegaly, fever and generalized lymphadenopathy. One was treated for non-Hodgkin's lymphoma and had previously been employed in Saudi Arabia ${ }^{3}$, while another was an elderly local. ${ }^{5}$ The third was a young, previously well female of European origin. ${ }^{6}$ The fourth was a 53-year-old farmer from Batticaloa, who had an accidental injury to the same area in the oral cavity two years prior caused 'by the stick used to drive the buffaloes while ploughing the fields'. ${ }^{2}$ The fifth was a 59 year old farmer from Akuressa, a poorly-controlled diabetic, betel chewer and illicit liquor consumer. ${ }^{7}$

Clinically, histoplasmosis may manifest as pulmonary or disseminated forms. The disseminated form manifests itself when haematogenous spread occurs through parasitized macrophages. Foci of viable organisms can remain in various organs, and get reactivated many years later, as they are not completely killed by the immune system. ${ }^{8}$

Certain patient groups are vulnerable to develop acute dissemination of the organism during periods of low cell mediated immunity. These include patients with haematological malignancies, transplant recipients, those on corticosteroids and tumour necrosis factor antagonists, HIV, infants and the elderly. These patients suffer from a slowly progressive and usually fatal form of the disease, unless effectively treated. ${ }^{8}$ It has been found that those over 55 years of age are at risk for disseminated infection. ${ }^{9}$ In our patient, the only risk factor of note was his age, as he was not suffering from any of the above conditions and HIV screening was negative.

Mucous membrane ulceration in the gingival and buccal mucosa or tongue may prompt the clinician to consider oral squamous cell carcinoma, as in our patient. Other sites involved in disseminated histoplasmosis may include lips, pharynx and larynx, well as skin ulcers and nodules. 
Almost any system can be affected. In any patient with mucocutaneous lesions due to histoplasmosis, disseminated infection should always be considered, as isolated oral lesions are very infrequent. ${ }^{8}$ We therefore presumed this patient was suffering from disseminated histoplasmosis, due to the presence of the oral lesions and asymptomatic granulomas in the chest. Chronic pulmonary histoplasmosis may present similarly to pulmonary tuberculosis. Most patients have pre-existing lung pathology such as chronic obstructive pulmonary disease. ${ }^{8}$

Histology and fungal cultures of biopsy samples are the gold standard for the diagnosis of histoplasmosis. In addition, antigen testing and serology are sensitive, have a shorter turn-aroundtime, and can be easily interpreted by the clinician. ${ }^{10} \mathrm{H}$. capsulatum antigen detection in serum and urine is useful, specially to monitor treatment response. ${ }^{8}$ Although molecular methods are available, none are FDA approved for routine clinical use as yet. ${ }^{10}$

\section{Conclusion}

Due to its uncommon nature, histoplasmosis is possibly under-reported in Sri Lanka. A high index of suspicion is needed, as the identification of this organism requires specialized laboratory tests and prolonged incubation.

Conflicts of interest: Authors declare that there are no conflicts of interest.

\section{References}

1. Histoplasma capsulatum (Histoplasmosis) George S. Deepe, Jr. In : Mandell, Douglas \& Benett's Principles and practice of infectious diseases. Eigth edition 2015. Volume 1. 2949-2962. Elsevier

2. Jayaweera FRB, Attapatu M, Fonseka I, et al. Histoplasmosis of the buccal cavity. Ceylon Med $J ; 1975 ; 20(1): 45-47$ No doi

3. Karunanayake L. Samaraweera I.P., de Silva K., et al. Isolation of Histoplasma capsulatum from an immunocompromised patient. Bulletin of the Annual Academic Sessions of the Kandy Society of Medicine (KSM). 2009; 31:188-189 No doi

4. Randhawa HS, Gugnani HC,. Occurrence of histoplasmosis in the Indian sub-continent: An overview and update. J Med Res Prac; 2018; 7(3):71-83 doi: https://doi.org/10.20936/jmrp/07/03/02

5. Sigera LSM., Gunawardane SR, Malkanthi MA, et al. Histoplasma capsulatum caused a localized tongue ulcer in a non-HIV patient - a case from nonendemic country. Ear, Nose \& Throat Journal 2019; Epub; 1-3. doi: 10.1177/0145561319844246.

6. Harten P, Euler HH, Wolf E, et al. Disseminated histoplasmosis in a non-immunocompromised host. Clin Investig. 1994; 72(11):878-882. doi: http://dx.doi.org/10.1007/BF00190745

7. Vidanagama D, Dias DK, Wijayaratne WMDGB, et al. Histoplasmosis of the oral cavity. Bull Sri Lanka College Microbiol. 2010; 8:27. No doi

8. Kauffman CA. Histoplasmosis: A clinical and laboratory update. Clin Microbiol Rev. 2007; 20(1):115132. doi: $h t t p: / / d x$.doi.org/10.1128/CMR.00027-06

9. Wheat LJ, Slama TG, Norton JA, et al. Risk factors for disseminated or fatal histoplasmosis. Analysis of a large urban outbreak. Ann Intern Med. 1982; 96(2):159-63. doi: http://dx.doi.org/10.7326/00034819-96-2-159

10. Azar MM, Hage CA. Laboratory diagnostics for histoplasmosis. J Clin Microbiol 2017; 55:1612-1620. doi: https://doi.org/10.1128/JCM .02430-16. 